\title{
Video Article \\ Cerebrovascular Casting of the Adult Mouse for 3D Imaging and Morphological Analysis
}

\author{
Espen J. Walker ${ }^{1}$, Fanxia Shen ${ }^{1}$, William L. Young ${ }^{1,2,3}$, Hua Su ${ }^{1}$ \\ ${ }^{1}$ Center for Cerebrovascular Research, Department of Anesthesia and Perioperative Care, University of California, San Francisco \\ ${ }^{2}$ Department of Neurological Surgery, University of California, San Francisco \\ ${ }^{3}$ Department of Neurology, University of California, San Francisco
}

URL: https://www.jove.com/video/2958

DOI: doi:10.3791/2958

Keywords: Neuroscience, Issue 57, vessel, vascular cast, capillary, cerebrovasculature, brain, blood, AVM, fistula

Date Published: 11/30/2011

Citation: Walker, E.J., Shen, F., Young, W.L., Su, H. Cerebrovascular Casting of the Adult Mouse for 3D Imaging and Morphological Analysis. J. Vis. Exp. (57), e2958, doi:10.3791/2958 (2011).

\section{Abstract}

Vascular imaging is crucial in the clinical diagnosis and management of cerebrovascular diseases, such as brain arteriovenous malformations (BAVMs). Animal models are necessary for studying the etiopathology and potential therapies of cerebrovascular diseases. Imaging the vasculature in large animals is relatively easy. However, developing vessel imaging methods of murine brain disease models is desirable due to the cost and availability of genetically-modified mouse lines. Imaging the murine cerebral vascular tree is a challenge. In humans and larger animals, the gold standard for assessing the angioarchitecture at the macrovascular (conductance) level is x-ray catheter contrast-based angiography, a method not suited for small rodents.

In this article, we present a method of cerebrovascular casting that produces a durable skeleton of the entire vascular bed, including arteries, veins, and capillaries that may be analyzed using many different modalities. Complete casting of the microvessels of the mouse cerebrovasculature can be difficult; however, these challenges are addressed in this step-by-step protocol. Through intracardial perfusion of the vascular casting material, all vessels of the body are casted. The brain can then be removed and clarified using the organic solvent methyl salicylate. Three dimensional imaging of the brain blood vessels can be visualized simply and inexpensively with any conventional brightfield microscope or dissecting microscope. The casted cerebrovasculature can also be imaged and quantified using micro-computed tomography $(\text { micro-CT })^{1}$. In addition, after being imaged, the casted brain can be embedded in paraffin for histological analysis.

The benefit of this vascular casting method as compared to other techniques is its broad adaptation to various analytic tools, including brightfield microscopic analysis, CT scanning due to the radiopaque characteristic of the material, as well as histological and immunohistochemical analysis. This efficient use of tissue can save animal usage and reduce costs. We have recently demonstrated application of this method to visualize the irregular blood vessels in a mouse model of adult BAVM at a microscopic level ${ }^{2}$, and provide additional images of the malformed vessels imaged by micro-CT scan. Although this method has drawbacks and may not be ideal for all types of analyses, it is a simple, practical technique that can be easily learned and widely applied to vascular casting of blood vessels throughout the body.

\section{Video Link}

The video component of this article can be found at https://www.jove.com/video/2958/

\section{Clearing Blood from Vasculature}

1. Anesthetize mouse by intraperitoneal injection of $100 \mathrm{mg} / \mathrm{kg}$ ketamine with $10 \mathrm{mg} / \mathrm{kg}$ xylazine diluted in $0.25 \mathrm{ml}$ saline.

2. Once anesthesia has been confirmed by no response to a paw pinch, use scissors to make an incision across the chest with scissors just below the xyphoid process, cut through diaphragm, and cut up between dorsal and ventral segment of ribcage to expose thoracic cavity. Expose heart by folding up sternum and adjacent chest wall and secure with hemostat. Open the pericardial sac with tweezers to expose the heart.

3. Turn on perfusion pump $\left(100 \mathrm{mmHg}\right.$ pressure) connected to warm PBS plus heparin $(5 \mathrm{unit} / \mathrm{ml})$ solution in hot water bath at $37^{\circ} \mathrm{C}$ and tubing with a blunt 22 gauge needle on the outflow.

4. Insert the needle into the left ventricle, near the apex of the heart. Immediately cut open the right atrium to enable systemic blood outflow and continue flushing until blood is removed from circulation. 


\section{Perfusion of Casting Agent into the Vascular System}

1. Prepare Microfil (Flow Tech, Inc. Carver, MA) casting solution per manufacturer's protocol: Mix $5 \mathrm{ml}$ of MV diluent with $4 \mathrm{ml}$ of filtered MV compound. Add $450 \mu \mathrm{l}(5 \%)$ of catalyst (MV curing agent). Solution working time is 20 minutes, but mix immediately before use to ensure proper viscosity.

2. Withdraw casting agent solution into a $10 \mathrm{ml}$ syringe, attach a blunt 20 gauge needle to the syringe and fill needle with solution.

3. Insert needle into the left ventricle through the same hole used for flushing the blood. Secure needle into place with hemostat clamp on the heart.

4. Inject casting agent solution slowly into the left ventricle at approximately $3 \mathrm{ml} / \mathrm{min}$. Excess pressure may lead to incorrect flow of casting agent or potential vessel rupture.

5. Look for signs of successful perfusion, including: visualization of casting agent in intestine and liver vasculature, distal limb discoloration, and nose and tongue discoloration. Readjust needle if necessary to achieve proper perfusion.

\section{Collection and Processing of Tissue}

1. Remove brain and place in $4 \%$ paraformaldehyde overnight at $4^{\circ} \mathrm{C}$.

2. Dehydrate brain by placing from paraformaldehyde directly into increasingly concentrated $\mathrm{EtOH}$ at room temperature: $25 \% \mathrm{EtOH}, 1$ day; $50 \%$ $\mathrm{EtOH}, 1$ day; $75 \% \mathrm{EtOH}, 1$ day; 95\% EtOH, 2 days; and $100 \% \mathrm{EtOH}, 2$ days.

3. Clarify brain tissue around blood vessels by immersing the perfused brain in methyl salicylate for 2 days at room temperature.

4. Vasculature of the whole unsectioned clarified brain can be imaged with brain immersed in methyl salicylate under a dissecting microscope (Fig. 1) (Leica MZFL III microscope, Leica Microsystems, Bannockburn, IL) or brightfield microscope (Fig. 2) (Leica DM/LS, Leica Microsystems).

5. Reclarification can be done if tissue is not completely cleared. From methyl salicylate, transfer brain to $95 \%$ EtOH for 2 days, $100 \%$ EtOH for 2 days, and methyl salicylate for 2 days.

\section{Micro-CT Imaging or Histological Application- Tissue Preparation}

1. The brain can be imaged using micro-CT directly after clarification or after re-hydration (Fig. 4).

2. To prepare brain tissue for histological analysis, rehydrate the brain through serial diluted $\mathrm{EtOH}: 100 \% \mathrm{EtOH}, 1$ day; $95 \% \mathrm{EtOH}, 1$ day; $75 \% \mathrm{EtOH}, 1$ day; $50 \% \mathrm{EtOH}, 2$ days; $25 \% \mathrm{EtOH}, 2$ days; and store in PBS. The brain can then be embedded in paraffin using a standard procedure.

\section{Representative Results}

Following vascular casting, the cerebrovasculature can be seen macroscopically on the brain surface (Fig.1A). The vascular tree inside the parenchyma can be visualized following clarification (Fig. 1B). The detailed vascular structure can be imaged and analyzed under a brightfield microscope (Fig. 2), dissecting scope (Fig. 3), and with micro-CT scanning (Fig. 4). Using brightfield microscopy, the vasculature can be viewed at different focal planes without sectioning the brain (Fig. 2A) as well as individual microvessels at higher magnification (Fig. 2B). Under a dissecting scope, one can see the entire vascular structure in one focal plane, allowing better visualization of the three dimensional structure of the cerebrovasculature (Fig. 3). Images taken under a dissecting microscope can show large vessel morphology and whole brain vasculature, but lack the details shown in pictures taken with brightfield microscopy (Fig. 2B). This technique has allowed us to detect irregular vessels in our experimental BAVM adult mouse model ${ }^{2}$. Figure 5 shows an example of the same irregular vessels detected by micro-CT scanning (Fig. $5 \mathrm{~A}$ ) and brightfield microscope (Fig. 5B). Together, this data shows that we can analyze the same sample with multiple imaging tools after vascular casting. 

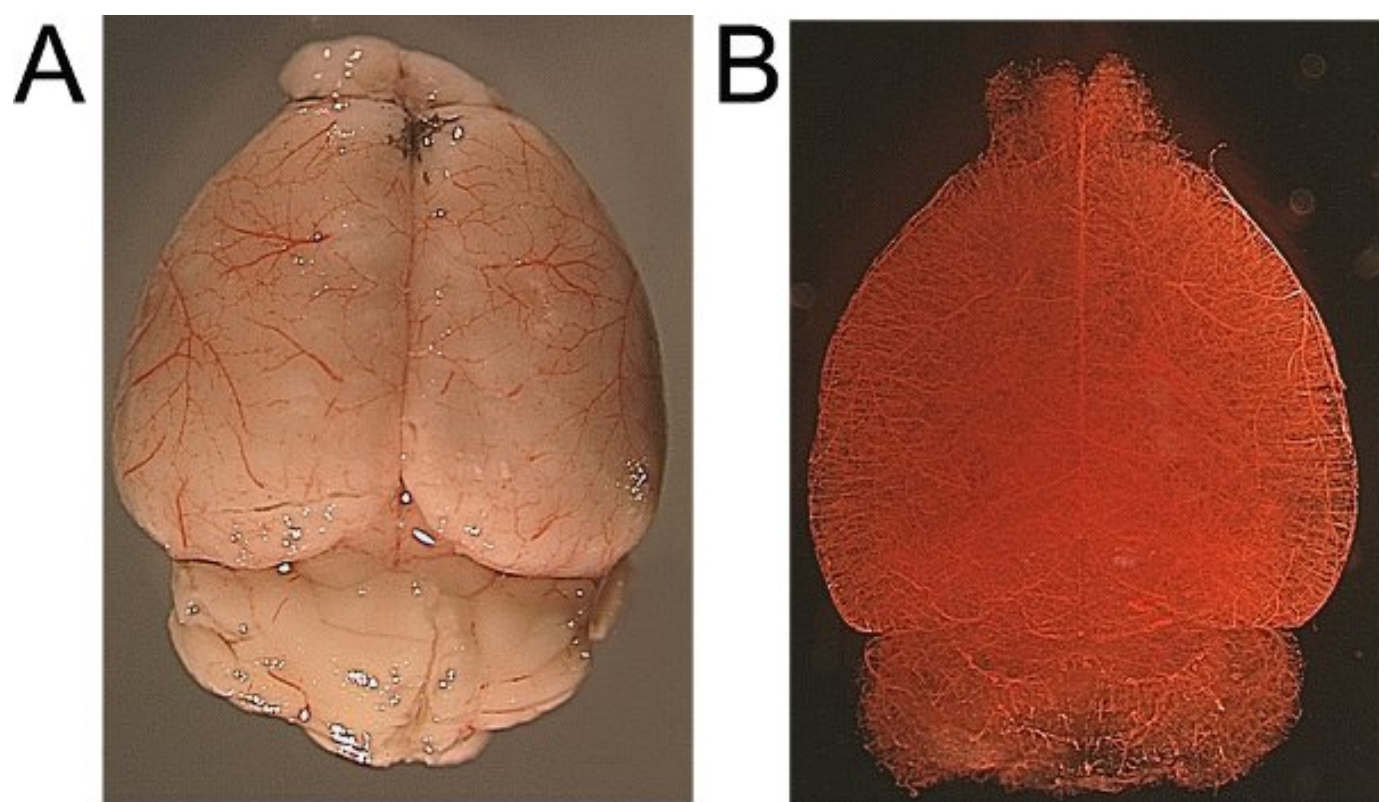

Figure 1. Whole mouse brain following vascular casting. Before $(A)$ and after (B) clarification by methyl salicylate solvent. Vessels can be clearly visualized on the brain surface $(A)$ and in parenchyma following clarification procedure $(B)$.

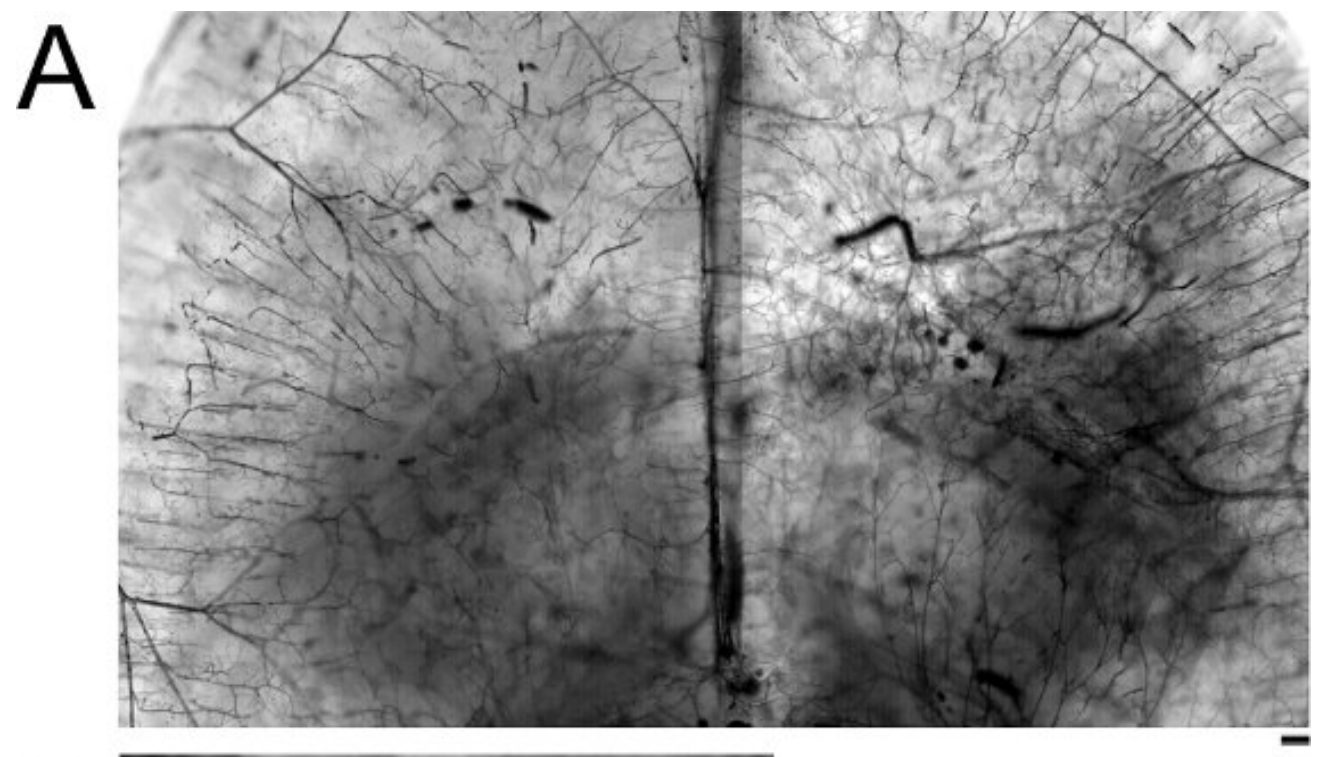

$\mathrm{B}$

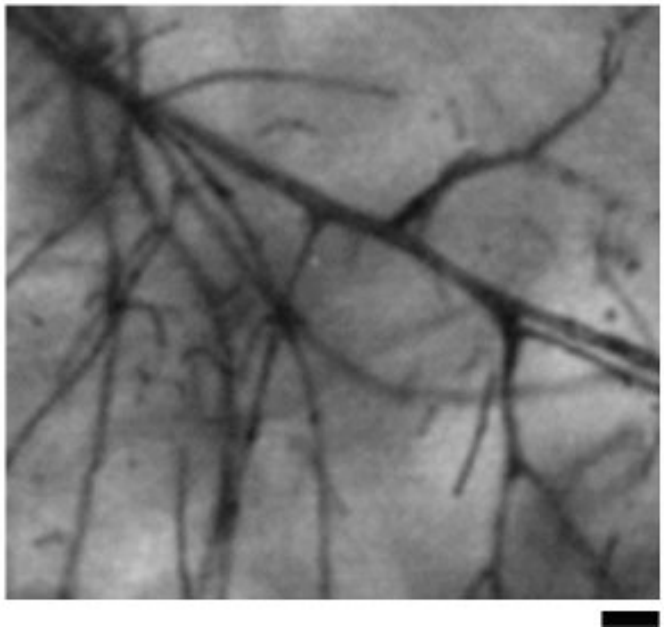


Figure 2. Brightfield microscope images of clarified vascular casted brain. Brain can be imaged at various planes and at different magnification levels. Scale Bar $200 \mu \mathrm{m}(\mathrm{A})$ and $50 \mu \mathrm{m}(\mathrm{B})$.

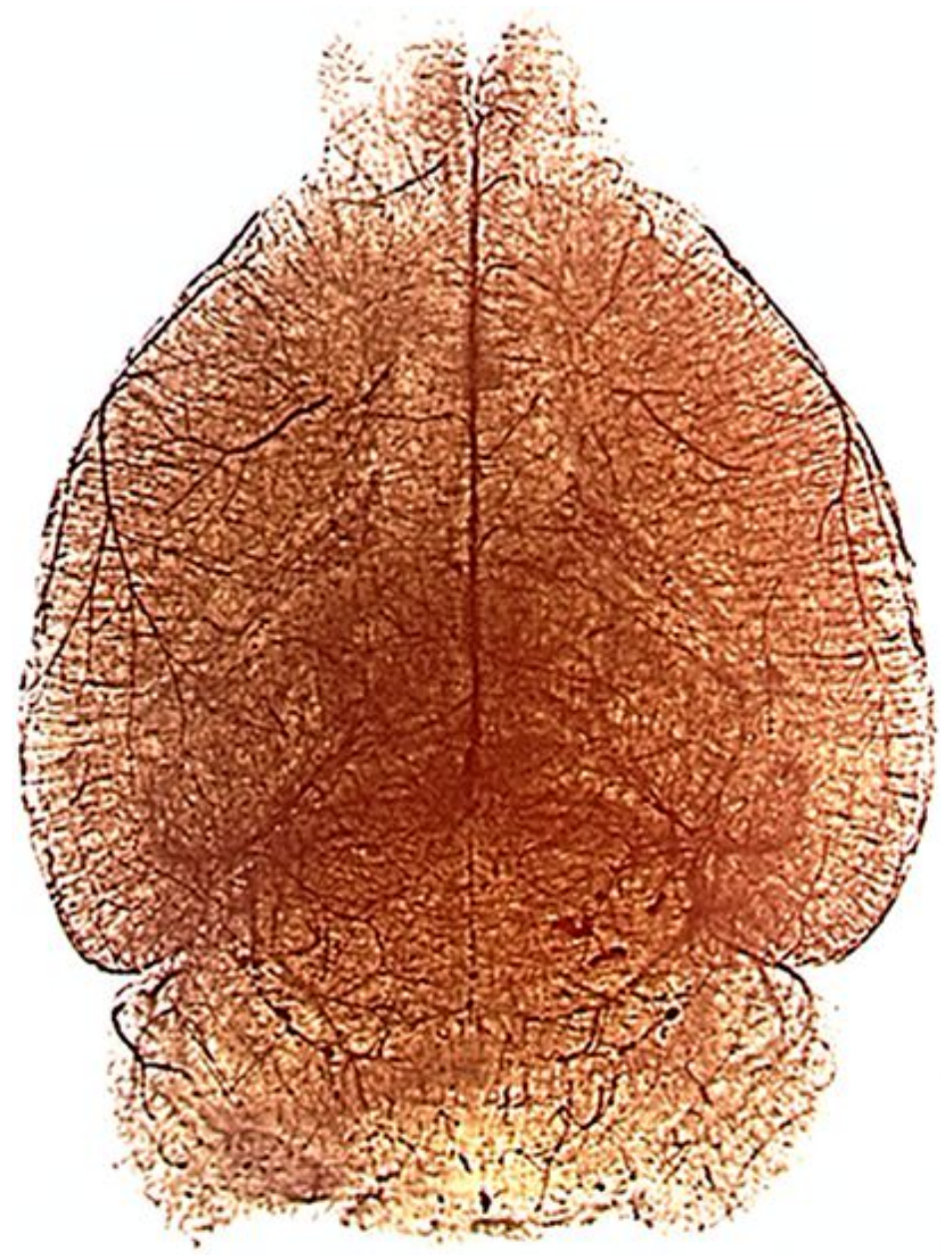

Figure 3. Dissecting microscope image at $1 \mathrm{x}$ showing whole adult mouse brain cerebrovasculature from a dorsal view. 


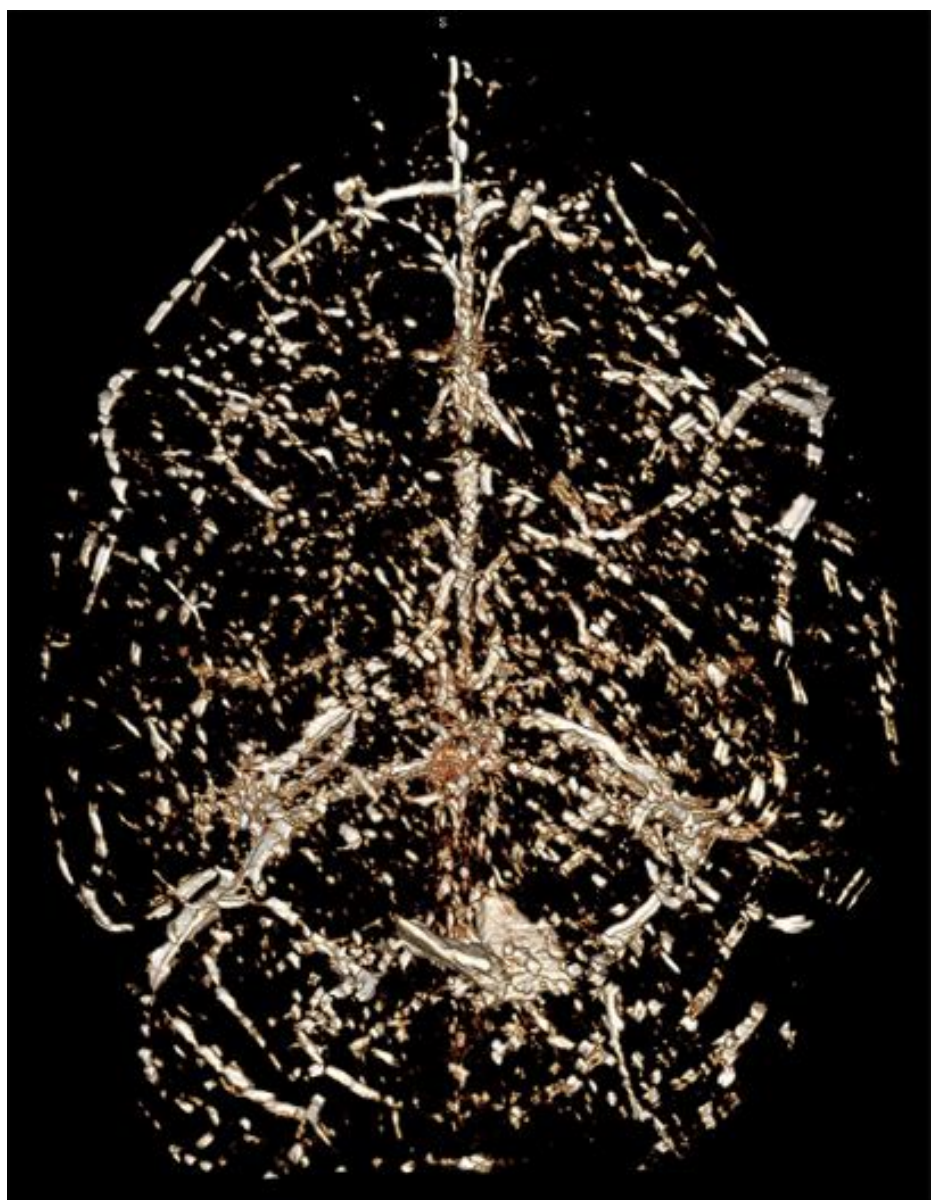

Figure 4. Micro-CT scanning image of the same whole adult mouse brain shown in Figure 3 from a dorsal view. 

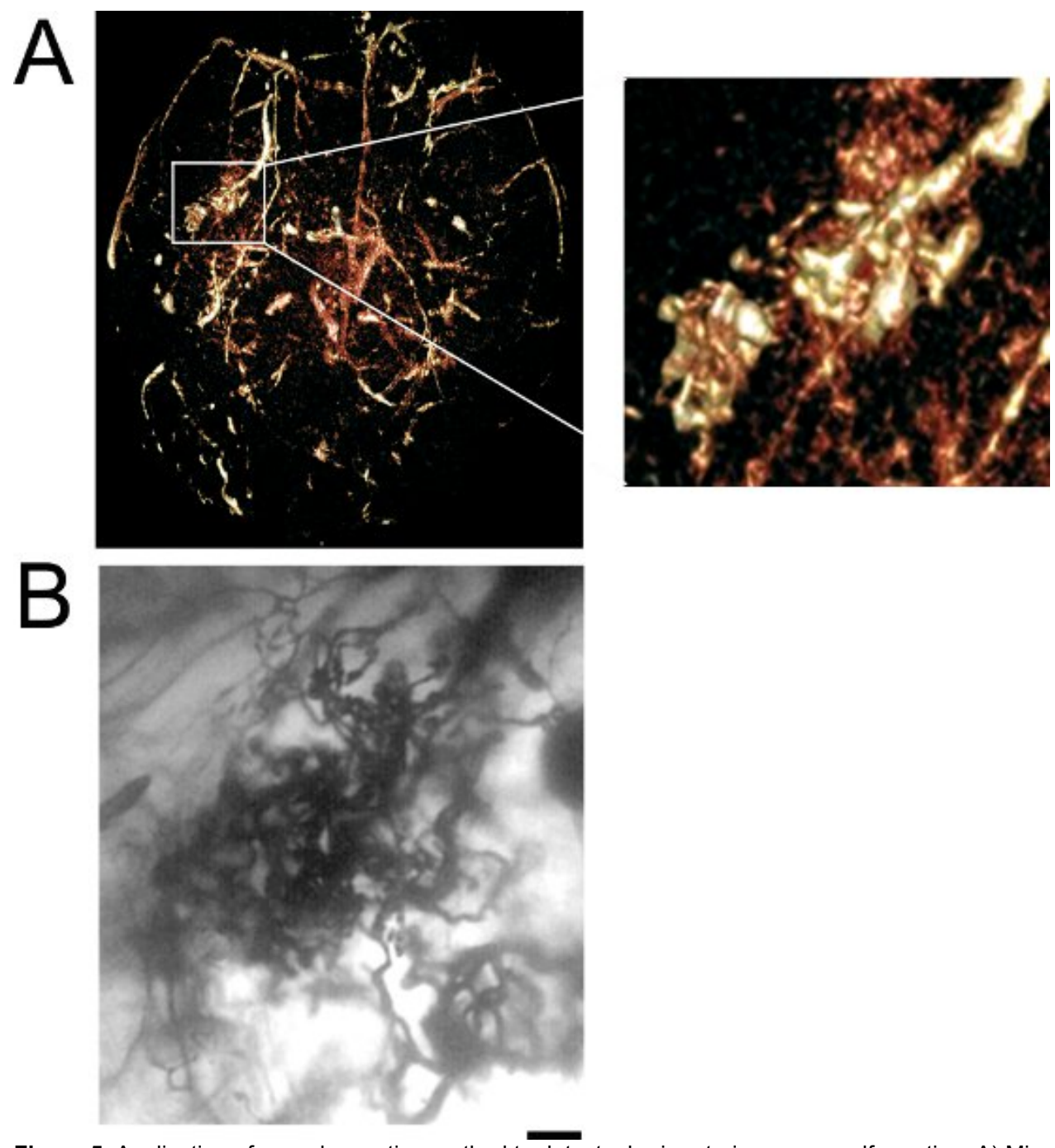

Figure 5. Application of vascular casting method to detect a brain arteriovenous malformation. A) Micro-CT scan of vascular casted brain shows region of enlarged, irregular AVM-like vessels in right hemisphere from a ventral view. Enlarged image of region (white box) is shown on the right. B) Same irregular vessels visualized under brightfield microscope at 50x magnification. Scale bar $50 \mu \mathrm{m}$.

\section{Discussion}

We report here a method for casting of adult mouse cerebrovasculature that may be imaged with various modalities. Application of this method to the disease model BAVM enables 3D analysis of irregular vessels. Potential future studies include quantification of micro-CT images, histological analysis of tissue, and diagnosis of vascular disease progression.

\section{Common Problems Encountered and Suggestions}

Complete perfusion of the brain vasculature is not always achieved. Having enough pressure to reach the distal blood vessels without rupturing the left atrium or aorta is a challenge. To overcome this, apply consistent pressure of approximately $100 \mathrm{mmHg}$. Also, adjust needle position to ensure appropriate outflow of casting agent into the aorta. It is important that the carotid arteries are not restricted so the casting agent can reach the brain vessels. Critical procedures that correlate with improved small vessel perfusion are: (1) proper clearing of the blood from the vasculature; (2) filtration of the casting agent; and (3) needle placement during casting agent injection. Care should be taken to avoid injection of casting agent into the left atrium and lung. Other investigators have successfully perfused mouse ${ }^{3}$ and rat ${ }^{4}$ cerebrovasculature without using additional procedures. Manual analysis of Microfil perfused vessels counterstained with hemotoxylin showed $93 \%$ vessel lumen perfusion ${ }^{5}$. Always discard or completely clean any tool that comes in contact with the casting agent as it will polymerize quickly, and tools cannot be used for multiple subjects. Clarification of the surrounding mouse brain tissue can be challenging. Following immersion in methyl salicylate, the tissue may still appear opaque, making vessel imaging difficult. Ensuring proper dehydration and clarification should solve this problem. Placing brain tissue on a rocker during alcohol and methyl salicylate treatment can improve tissue clarification.

\section{Drawbacks of the Technique}

The limitations of this technique include: (1) it is necessary to inject the casting agent solution immediately after mixing it as it will thicken quickly after the catalyst is added; (2) the casting agent fills large conductance vessels easily, but it does not reliably fill smaller vessels, such 
as arterioles (resistance vessels) and capillaries (nutritive vessels); and (3) it is not the best radiopaque contrast for micro-CT imaging, although some groups prefer Microfil for micro-CT imaging ${ }^{1,6}$. However, following the suggestions proposed above, including proper needle placement, vessel position and perfusion pressure, complete casting of smaller vessels can be achieved. The adaptability to various analysis tools makes this agent a valuable tool for analyzing cerebrovascular structure, including arterial and venous circulation, with various perspectives.

\section{Applications of the Technique}

The benefit of this method is its broad application potential. The cast fills all vessels, including arteries, veins, and capillaries of all tissue within the body, so vascular structures in multiple organs of various species can be analyzed. Previously, other groups have used this perfusion for large $^{7}$ and small ${ }^{8}$ vessels in human, monkey ${ }^{9}$, sheep ${ }^{10}$, rat ${ }^{4,11,12}$, and mouse ${ }^{3,13}$. We now show evidence of using this technique in analyzing the structure of microvessels in the mouse brain, in both healthy and diseased states ${ }^{2}$. In addition, there are Microfil solutions with various viscosities available to perfuse vessels of different sizes and different colors to improve the visualization of the vessels in various organs. For example, red can be easily visualized in the pale brain parenchyma, and yellow contrasts well with dark red myocardium. Moreover, as the material is radiopaque, vasculature can be imaged with micro-CT scanning to obtain a 3D rotatable image. The tissue can also be used for paraffin sections and histological analysis after being imaged.

\section{Conclusion}

We demonstrate here a protocol to make a vascular cast of the blood vessels of the adult mouse brain, which can be adapted using a variety of analysis techniques to study the morphological structure of the cerebrovasculature. We have also provided evidence for the application of this method to a model of the BAVM disease state, represented by enlarged, irregularly shaped vessels that shunt blood from arterial to venous circulations. There are a variety of vascular casting methods available. The simple protocol we provide here for a vascular cast may be used as a tool to examine the vasculature of the adult mouse brain using various imaging modalities.

\section{Disclosures}

Experimental procedures for using laboratory animals were approved by the Institutional Animal Care and Use Committee of the University of California, San Francisco (UCSF).

\section{Acknowledgements}

The authors thank Voltaire Gungab for assistance with manuscript preparation. This work was supported in part by grants from: the National Institutes of Health (T32 GM008440 to E.J. Walker, R01 NS27713 to W.L. Young, P01 NS44155 to W.L. Young and H. Su), and R21 NS070153 to $\mathrm{H}$. Su; and the American Heart Association (AHA 10GRNT3130004 to H. Su).

\section{References}

1. Bolland, B.J., Kanczler, J.M., Dunlop, D.G., \& Oreffo, R.O. Development of in vivo muCT evaluation of neovascularisation in tissue engineered bone constructs. Bone. 43, 195-202 (2008).

2. Walker, E.J., et al. Arteriovenous malformation in the adult mouse brain resembling the human disease. Ann. Neurol. (Epub ahead of print) (2011).

3. Iqbal, U., et al. Kinetic analysis of novel mono- and multivalent $\mathrm{VHH}$-fragments and their application for molecular imaging of brain tumours. Br. J. Pharmacol. 160, 1016-1028 (2010).

4. Fukunaga, A., Kawase, T., \& Uchida, K. Functional recovery after simultaneous transplantation with neuro-epithelial stem cells and adjacent mesenchymal tissues into infarcted rat brain. Acta. Neurochir. (Wien). 145, 473-480 (2003).

5. Chugh, B. P., et al. Measurement of cerebral blood volume in mouse brain regions using micro-computed tomography. Neuroimage. 47, 1312-1318 (2009).

6. Marxen, M., et al. MicroCT scanner performance and considerations for vascular specimen imaging. Med. Phys. 31, 305-313 (2004).

7. Barger, A. C., Beeuwkes, R., 3rd, Lainey, L. L. \& Silverman, K. J. Hypothesis: vasa vasorum and neovascularization of human coronary arteries. A possible role in the pathophysiology of atherosclerosis. N. Engl. J. Med. 310, 175-177 (1984).

8. Fossett, D.T. \& Caputy, A.J. Operative Neurosurgical Anatomy (Thieme Medical Publishers, New York, 2002).

9. Reynolds, D.G., Brim, J., \& Sheehy, T.W. The vascular architecture of the small intestinal mucosa of the monkey (Macaca mulatta). Anat. Rec. 159, 211-218 (1967).

10. Bernard, S., Luchtel, D.L., Polissar, N., Hlastala, M.P., \& Lakshminarayan, S. Structure and size of bronchopulmonary anastomoses in sheep lung. Anat. Rec. A. Discov. Mol. Cell. Evol. Biol. 286, 804-813 (2005).

11. Brady, J.M. \& Cutright, D.E. A new technique of measuring blood vessel volume in bone applied to the mandible and humerus of the rat. Anat. Rec. 170, 143-146 (1971).

12. Evan, A.P. \& Dail, W.G., Jr. Efferent arterioles in the cortex of the rat kidney. Anat. Rec. 187, 135-145 (1977).

13. Murphy, P.A., et al. Endothelial Notch4 signaling induces hallmarks of brain arteriovenous malformations in mice. Proc. Natl. Acad. Sci. U. S. A. 105, 10901-10906 (2008). 